\title{
Biomedicalización de los tiempos: terapéuticas, biosocialidades y cuidados cotidianos en familias de niños y niñas con diagnóstico de TEA en Buenos Aires
}

Biomedicalization of times: therapies, biosocialities and daily cares in children with ASD diagnostic in Buenos Aires

\section{Eugenia Bianchi ${ }^{\mathrm{a}}$}

(D) https://orcid.org/0000-0003-2311-7490

E-mail: eugenia.bianchiळgmail.com

\section{Stephany Rodríguez Juradob}

(D) https://orcid.org/0000-0001-8559-0545

E-mail: stephany.rodriguezjurado®gmail.com

anniversidad de Buenos Aires. Facultad de Ciencias Sociales. Consejo Nacional de Investigaciones Científicas y Técnicas. Buenos Aires, Bs. As., Argentina.

bUniversidad de Buenos Aires. Facultad de Derecho. Ciudad de Buenos Aires, Bs. As., Argentina.

\section{Correspondencia}

Eugenia Bianchi

Pres. José Evaristo Uriburu, 950, 6to. piso. Buenos Aires, Argentina. Cp III4.

\section{Resumen}

Se analiza la incidencia del diagnóstico de Trastorno del Espectro Autista (TEA) sobre la percepción y organización del tiempo en la vida cotidiana, de parte de las familias, y en especial las madres de niños y niñas diagnosticados por TEA (NNDxTEA). Los resultados surgen de dos investigaciones de posgrado. La metodología es cualitativa, reconstruye las historias de vida de cuatro madres que durante 2015 acompañaron a sus NNDxTEA a un Centro Terapéutico en la Provincia de Buenos Aires, Argentina. Se consideran dos líneas. Primera, la medicalización y biomedicalización como procesos de anillados concéntricos en tres niveles: macro, meso y micro, profundizando el análisis en este último. Segunda, la vinculación entre los procesos de diagnóstico y tratamiento en salud mental, y las biosocialidades. Adicionalmente se incorporan consideraciones desde la sociología del tiempo. Los resultados exponen la incidencia del diagnóstico de TEA sobre el tiempo en cuatro ejes: la transformación de las actividades cotidianas, por la asistencia a espacios terapéuticos; las expectativas respecto del tiempo libre y esparcimiento; los cambios que, de haber podido, hubieran hecho en el tiempo que los NNDxTEA llevan realizando las terapias; y las creencias y pensamientos acerca del futuro del NNDxTEA de parte de sus familiares.

Palabras clave: TEA; Medicalización; Biomedicalización; Biosocialidades; Cuidados. 
The influence of the Autistic Spectrum Disorder (ASD) diagnosis on the perception and organization of time in daily life by families, especially mothers, of children diagnosed with ASD is analyzed. Results stem from two graduate investigations. The methodology is qualitative, reconstructing the life stories of four mothers who, throughout 2015, accompanied their children diagnosed with ASD to a Therapeutic Center in the Province of Buenos Aires, Argentina. Two lines are considered: first, the medicalization and biomedicalization as concentric ringed processes in three levels: macro, meso and micro, deepening the analysis in the latter. Second, the link between diagnostic and treatment processes in mental health and the biosocialities. Some considerations on sociology of time are also incorporated. The results show the incidence of the ASD diagnosis over time, in four areas: the transformation of daily activities, by attending therapeutic spaces; the expectations regarding free time and leisure; the changes that, if possible, have been made in the time the children diagnosed with ASD have been carrying out the therapy; and the beliefs and thoughts of their relatives about the future of the children diagnosed with ASD.

Keywords: ASD; Medicalization; Biomedicalization; Biosocialities; Caring.
Este artículo analiza la incidencia del diagnóstico de Trastorno del Espectro Autista (TEA) sobre la percepción y organización del tiempo en la vida cotidiana, de parte de las familias, y en especial las madres, de los niños y niñas que recibieron este diagnóstico. Se exponen resultados de un estudio realizado en 2015 en un Centro Terapéutico en la provincia de Buenos Aires, Argentina, al que asistieron niños y niñas diagnosticados por TEA (en adelante NNDxTEA), y algunos de sus familiares. El resultado proviene de una sistematización conjunta de dos investigaciones de posgrado, realizando una relectura de las fuentes en una clave no explorada previamente, y que dio lugar a nuevas líneas de análisis. Los datos fueron obtenidos de acuerdo a una metodología cualitativa, reconstruyendo las historias de vida de cuatro madres de NNDxTEA que asistieron al antedicho centro terapéutico.

Se consideran dos líneas de los estudios de la medicalización y biomedicalización de la sociedad. En primer lugar, la medicalización y biomedicalización como procesos de anillados concéntricos en tres niveles o registros: macro, meso y micro, profundizando el análisis en este último. En segundo lugar, la vinculación entre los procesos de diagnóstico y tratamiento en salud mental, y la creación o reformulación de biosocialidades. Adicionalmente se incorporan consideraciones desde la sociología del tiempo.

Los resultados exponen la incidencia del diagnóstico de TEA sobre el tiempo en cuatro ejes: La transformación de las actividades cotidianas, en virtud de la asistencia a espacios terapéuticos; Las expectativas respecto del tiempo libre y las actividades de esparcimiento; Los cambios que, de haber podido, hubieran realizado en el tiempo que los NNDxTEA llevan asistiendo a las terapias; y Las creencias y pensamientos acerca del futuro del NNDxTEA de parte de sus familiares.

\section{Trastorno del espectro autista y (bio) medicalización}

El TEA es definido por el Manual de Diagnóstico y Estadísticas de los Trastornos Mentales (DSM) 
como uno de los trastornos en el neurodesarrollo. Es mayormente diagnosticado en la infancia e incluye diversas características en aspectos cognitivos, socioeducativos y emocionales. En la actualidad, el TEA se define como una alteración en la comunicación y la interacción social, asociada a actividades e intereses restrictivos y repetitivos; además, puede estar acompañado, o no, de otros déficits cognitivos, sensoriales o médicos (APA, 2013).

Como nosología, el TEA ha sufrido diversas transformaciones. Fue descrito formalmente por primera vez por Leo Kanner en 1943, bajo el término de “autismo infantil temprano", y caracterizado por una "extrema soledad, alteraciones en el lenguaje y en la flexibilidad" (Kanner, 1993). En 1944, Hans Asperger denominó "psicopatía autista” a un cuadro clínico con características similares a las descritas por Kanner (Asperger, 1944 apud Frith, 1992).

El cambio más reciente en la clasificación del TEA está expresado en el DSM-5, la última versión del manual, que tipifica al autismo en el marco de los Trastornos del Espectro Autista (TEA), y subraya dos categorías: Trastorno de la Comunicación Social, y Patrones repetitivos de Comportamiento, Intereses y Actividades. Los criterios diagnósticos abarcan antecedentes conductuales y comportamientos actuales. El nivel de severidad ocupa un lugar destacado, englobando como dificultades del desarrollo las limitaciones sociales y de la comunicación, y la presencia de patrones comportamentales repetitivos y restringidos (APA, 2013).

Dicho cambio ha generado diferentes posturas. Algunas indican que la nueva clasificación puede ser imprecisa e incidir en el número de casos diagnosticados con TEA, ya que no se limita al cuadro clásico descrito por Kanner (Paris, 2013). También que, en el momento del diagnóstico, los profesionales pueden tener dudas de su dictamen, especialmente en casos en los que las alteraciones son leves o se observan en edades prematuras (Valentine, 2010).

El aumento progresivo y la generación de nuevos diagnósticos se asocian a procesos de medicalización de la sociedad y de la infancia (Conrad; Potter, 2003), una corriente crítica de análisis que lleva casi setenta años de numerosas contribuciones
(Clarke; Shim, 2011). Sea que se la considere un área de estudios (Conrad, 2013) o se le otorgue el rango de teoría (Clarke et al., 2010), los abordajes de los fenómenos que "se vuelven médicos" (Conrad, 2007, p. 5) ocupan hoy un lugar relevante, diversificando y ampliando su alcance más allá de la sociología médica, para incluir disciplinas como historia, antropología, salud pública, economía y bioética (Conrad, 2013), y más recientemente, a los estudios sobre ciencia y tecnología, género, estratificación social y gubernamentalidad (Clarke et al., 2010).

A la vez la perspectiva se complejizó gracias a un conjunto de estudios en expansión acerca de la medicina y la salud, dando origen a diferentes especializaciones y afinamientos conceptuales; entre ellos, la biomedicalización (Bell; Figert, 2015; Clarke et al., 2010).

\section{Acerca de la micro-(bio) medicalización, las biosocialidades y la sociología del tiempo}

De los diferentes matices analíticos que habilita este amplio campo de estudios, para este artículo consideramos una línea procedente de un planteo iniciado hace casi cuarenta años por Conrad y Schneider (1980), continuado y complejizado por Halfmann (2012) y retomado por Clarke et al. (2010) en su caracterización de la biomedicalización. Si bien el lugar de los familiares no está explicitado en estos esquemas, consideramos que los autores plantean un conjunto de conceptos y argumentos, que son válidos para reflexionar en torno a las vivencias acerca de los tiempos cotidianos en los familiares de NNDxTEA. Con esto buscamos aportar a un área de vacancia en los estudios que, desde la medicalización y biomedicalización, abordan los procesos de diagnóstico y tratamiento en salud mental infantil.

Conrad y Schneider (1980) elaboraron una caracterización de los niveles de la medicalización, en un breve artículo que argumenta a favor de una tipología que refleje la complejidad del fenómeno. Esquemáticamente, proponen una estructura analítica en tres niveles: conceptual, institucional u organizacional, y de interacción entre médico 
y paciente. La relación entre estos niveles puede entenderse como un esquema de anillados concéntricos. En el primer, ubican actores como gobiernos, organizaciones nacionales y revistas e investigaciones médicas. En este nivel, el uso de un vocabulario o modelo médico apunta a la definición del problema, y no está centrado en los profesionales ni en los tratamientos. En el segundo, se congregan actores pertenecientes a las organizaciones locales. El rol de los médicos es formal, se limita a ser guardianes de los beneficios estatales, a través de la legitimación de una definición médica de un problema, pero no necesariamente actúan como ejecutores de tratamientos. Y en el tercer se hallan las interacciones médico-paciente, en las que a través de un diagnóstico el médico define un problema como médico y/o lo trata de alguna forma médica.

Aunque no es expresado en estos términos por los autores, estos niveles se corresponden con la división en macro, meso y micro que retoma Halfmann. En línea con lo expuesto por Conrad (2013) y Clarke et al. (2010), Halfmann resalta a la medicalización como un proceso que se manifiesta en gradientes, y desarrolla un esquema analítico en el que combina estos tres niveles con las dimensiones de los discursos, las prácticas y las identidades. Esta vinculación es la que nos interesa incorporar al análisis en este artículo.

Desde la teoría de la biomedicalización también se ha atendido a la relevancia de una caracterización de los fenómenos en términos macro, meso y micro. En el escrito fundacional de esta corriente, se subraya la incidencia de las transformaciones en las tecnologías biomédicas sobre la formación o reconfiguración de biosocialidades (Clarke et al., 2010). También destacan el carácter complejo de la medicalización y coinciden en identificarla como un proceso de gran magnitud, pero resaltan que no es monolítico, unidireccional ni inexorable, y que la contingencia es una de sus características más destacadas. También avanzan en destacar que la disponibilidad y masiva accesibilidad a tecnologías biológicas, incluyendo medicamentos, instrumentos diagnósticos y otros equipamientos, así como el acceso a enormes cantidades de información sobre enfermedades y trastornos (nuevos, antiguos y redefinidos) contribuyen a la creación de nuevas subjetividades, identidades y biosocialidades (Clarke et al., 2010).

La noción de biosocialidad fue acuñada por Rabinow y expresa nuevas formas de grupos sociales, comunidades e identidades conformadas en torno a un cierto rasgo biológico, como puede ser una variación genética específica o una clasificación biomédica (Rabinow, 1996). En particular, la biomedicalización conduce a la transformación de los cuerpos y a la creación de nuevas identidades, individuales o colectivas. En la creación de estas identidades, las tecnologías biomédicas cumplen un papel central, dado que impregnan numerosos aspectos de la vida cotidiana y los modos en que se viven las experiencias respecto de la salud y la enfermedad. Esto contribuye a la creación de subjetividades biomedicalizadas y biosocialidades. Las mismas pueden ser nuevas, o resultar de las aplicaciones tecnocientíficas a los cuerpos, que habilitan nuevos modos de acceder y desempeñarse en las identidades existentes. Además, existen diversos modos en los que la tecnociencia biomédica se involucra en los procesos de formación de identidades (Clarke et al., 2010).

Finalmente, la sociología del tiempo aporta una clave adicional de análisis para este artículo como una perspectiva que permite enfocar más allá del análisis centrado en la disciplina y lo institucional, y jerarquiza el estudio de las prácticas diarias (Zerubavel, 2004). Nos interesa trabajar los vínculos con la biomedicalización, en tanto proceso que incide y modela las actividades cotidianas, los cuidados y las nociones de salud y tratamientos, y con ello redunda en la conformación de subjetividades y biosocialidades específicas.

En particular, el estudio de Zerubavel (1981) fundamentó que los sistemas de calendarios y cronogramas tienen una función simbólica que excede los aspectos físico-matemáticos de su estructura, y los constituye como una entidad imbuida de sentidos sociales. Un punto de conexión entre esta posición y la perspectiva de la biomedicalización reside en la relevancia en común otorgada al carácter racional de la cultura moderna, y su introducción en la vida cotidiana. Zerubavel analiza este proceso desde la racionalización de aspectos como la precisión, la puntualidad, lo 
calculable, la estandarización, la burocratización, la rigidez, lo invariante, la coordinación y la rutina, como ejemplos de concentraciones altamente racionalizadas de órdenes temporales. En una línea afín aunque no específicamente dentro de la sociología del tiempo, Flaherty (1999) sostiene que la temporalidad es una faceta de la subjetividad y que está modelada por la sociedad e incidida por la socialización, una consideración consonante con los postulados que desde la biomedicalización sostienen que la biomedicina impone nuevos mandatos y desempeños (performances) que se incorporan al sentido del self. De esos desempeños emergen subjetividades acerca de lo que es saludable (y cabe agregar, también deseable y moralmente correcto) tanto al nivel de identidades subjetivas como de relaciones sociales (Clarke et al., 2010).

\section{Metodología}

En este artículo convergen dos investigaciones de posgrado. Ambas analizaron el actual diagnóstico de TEA desde la perspectiva de la biomedicalización de la sociedad, aunque con objetivos específicos diferentes. Sin embargo, como resultado de una relectura conjunta de las fuentes en una clave no explorada previamente, en este escrito los resultados fueron reconsiderados para jerarquizar la dimensión del tiempo como un organizador analítico de las experiencias de madres y familiares de NNDxTEA.

Las entrevistas que conforman las fuentes primarias se obtuvieron de acuerdo a una metodología cualitativa, siguiendo técnicas de entrevistas en profundidad de tipo semiestructurado (Marradi; Archenti; Piovani, 2007; Valles, 200o) para que se registren las historias de vida de cuatro madres de niños y niñas, que recibieron un diagnóstico de TEA y fueron tratados en un Centro Terapéutico en la provincia de Buenos Aires durante 2015. El Centro Terapéutico se especializa en la atención a población infantil con diagnósticos como TEA y Trastorno Generalizado del Desarrollo (TGD), desde una perspectiva neurocognitiva, y con especialistas en psicología, fonoaudiología, terapia ocupacional, psicopedagogía y neuropsicología.

La historia de vida permite realizar una aproximación a la realidad de las personas y su interpretación (Sandoval, 2002), y promueve dar cuenta de la "esencia subjetiva de la persona" a partir de las experiencias de vida en general, aunque remarcando en "los aspectos más importantes" (Atkinson, 1998, p. 28 apud Mallimachi; Giménez Véliveau, 2006, p. 196). Asimismo, como técnica de recolección de datos se orienta a recuperar momentos específicos de la biografía del entrevistado (Hernández Sampieri; Fernández-Collado; Baptista Lucio, 2006; Sarabia, 1985 apud Valles, 200o), asumiendo que todos los seres humanos expresan su pertenencia social y cultural a través de sus experiencias, y que por eso el investigador puede analizar el relato de los hechos de la vida de un individuo (Mallimachi; Giménez Véliveau, 2006).

En las entrevistas se jerarquizó que las madres relataran las biografías de los NNDxTEAy se enfatizó en sus propias experiencias respecto a los procesos médicos y terapéuticos frente al diagnóstico y tratamiento del TEA, y a sus vivencias y organización de la vida cotidiana. Estas aproximaciones temáticas se encuentran en consonancia con la historia de vida como herramienta metodológica, en tanto "la biografía de un individuo o la trayectoria de una familia se realizan a fin de profundizar en las preguntas que el investigador formula" (Mallimachi; Giménez Véliveau, 2006, p. 184).

Los criterios de selección de las participantes consistieron en: (1) que fueran madres de NNDxTEA o autismo según la valoración neuropsicológica emitida por el Centro Terapéutico, y que este diagnóstico hubiese sido dado entre los o y los 71 meses de edad; (2) que el niño o la niña hubiese asistido al centro terapéutico durante al menos un año de manera continua, previamente a la recolección de datos; (3) que al inicio del tratamiento el niño o la niña contaran con entre o y 5 años de edad; y (4) que el niño o la niña tuviesen al menos un/una hermano/a con quien convivieran. Una vez ubicados los casos que cumplían los criterios, se seleccionaron aleatoriamente cuatro participantes.

Se realizaron entrevistas en profundidad semiestructuradas (Marradi; Archenti; Piovani, 2007; Valles, 2000), en un marco de flexibilidad y espontaneidad en el relato. Las entrevistas se orientaron por una guía con cuatro ejes temáticos: datos personales acerca de la niña o el niño y su 
familia, primeras etapas e inicio del recorrido clínico, situación cotidiana en familia en la actualidad y procesos de cuidado, y crianza, vivencias y sentires respecto a la cotidianidad. Se incluyeron preguntas espontáneas que surgieron durante el desarrollo de la entrevista.

Las entrevistas se realizaron entre agosto y noviembre de 2015, mientras los NNDxTEA asistían a sesiones terapéuticas. Esta organización obedeció a la escasez de otros momentos disponibles de las madres. Las entrevistas se llevaron a cabo en espacios externos y cercanos a la institución (restaurantes, plazas o cafés), a fin de propiciar la comodidad de las madres y reducir el condicionante derivado del hecho de conversar en la institución. La toma de entrevistas requirió entre una y tres sesiones, con duraciones de entre 60 y 90 minutos en promedio cada una. Algunas participantes interrumpieron la entrevista para retornar al Centro y ocuparse de algunos eventos (por ejemplo, cambiar de pañal al NNDxTEA), para luego retomarla. Por tales motivos, en ocasiones las entrevistas fueron reprogramadas.

Aunque la convocatoria para la realización de las entrevistas fue abierta a las familias, mayoritariamente asistieron las madres. En algunos casos, sin embargo, las madres estuvieron acompañadas por otros hijos o hijas, o su pareja. Esto último sólo ocurrió en un caso, en una de las sesiones, y durante un corto período de tiempo. En la Tabla 1 sintetizan algunos datos de cada participante.

Las entrevistas fueron grabadas en audio, transcriptas y codificadas, y el análisis se realizó en un marco flexible e intuitivo. Los datos fueron identificados, segmentados y clasificados, a fin de disponer de ellos para su comprensión, y plantear resultados y conclusiones. Este proceso, realizado de un modo no secuencial sino simultáneo y recursivo, posibilitó la emergencia de nuevos significados sobre la temática abordada.

Las participantes del estudio lo hicieron en forma voluntaria, sin contraprestación económica, y autorizaron el registro sonoro y la toma de notas escritas de las entrevistas, mediante un consentimiento informado escrito y fechado, leído y firmado por las partes, reservando un ejemplar para cada una. La Resolución 1480/2011 del Ministerio de Salud de la República Argentina ofició de documento estructurador para confeccionar el consentimiento informado (Argentina, 2011). Los nombres de las participantes, de terceros y del Centro Terapéutico fueron modificados u omitidos, siguiendo criterios que garantizan la privacidad, confidencialidad y anonimato de la información. La participación en el estudio no supuso ningún riesgo para las madres.

Tabla I - Informaciones sobre cada participante

\begin{tabular}{|c|c|c|c|c|}
\hline & $\mathrm{Y}$, madre de $\mathrm{A}$ & $\mathrm{V}$, madre de $\mathrm{B}$ & L, madre de I & $M$, madre de $T$ \\
\hline $\begin{array}{l}\text { Edad al realizar las } \\
\text { entrevistas }\end{array}$ & 28 años & 33 años & 35 años & 37 años \\
\hline Estado Civil & Soltera & Casada & Unión libre & Casada \\
\hline Ocupación & $\begin{array}{l}\text { Cuidadora tiempo } \\
\text { completo }\end{array}$ & Idem & Idem & Idem \\
\hline $\begin{array}{l}\text { Edad del NNDXTEA al } \\
\text { realizar las entrevistas }\end{array}$ & 7 años & 5 años & 7 años & 4 años \\
\hline $\begin{array}{l}\text { Edad NNDXTEA al realizar } \\
\text { el diagnóstico }\end{array}$ & 3 años & 2 años, 8 meses & 3 años, I mes & $\begin{array}{c}\text { I año, } 8 \text { meses } \\
\text { (diagnóstico presuntivo) } \\
3 \text { años } \\
\text { (confirmación del diagnóstico) }\end{array}$ \\
\hline $\begin{array}{l}\text { Orden de nacimiento del } \\
\text { NNDXTEA }\end{array}$ & Primero & Primera & Tercero & Tercero \\
\hline $\begin{array}{l}\text { Edad de los demás hijos } \\
\text { e hijas }\end{array}$ & $\begin{array}{l}\text { Un hijo de } 6 \text { años; } \\
\text { una hija de I año }\end{array}$ & Un hijo de 4 años & $\begin{array}{c}\text { Dos hijas de } 9 \\
\text { y } 12 \text { años }\end{array}$ & Dos hijas de 12 y 14 años \\
\hline
\end{tabular}


Asimismo, durante el proceso de obtención de la información se tomaron notas en un diario de campo, consignando datos de las entrevistas, y otros datos verbales obtenidos de manera informal; así como también información relacionada con el análisis e interpretaciones luego de la recolección de los datos (Flick, 2004). Estas fuentes secundarias constituyen los "documentos de vida" (Plummer, 1983, p. 13 apud Mallimachi; Giménez Véliveau, 2006, p. 185), que robustecen la información obtenida en las entrevistas en profundidad.

\section{Resultados}

Exponemos a continuación las categorías resultantes, en cuatro ejes: (1) transformación de las actividades cotidianas, en virtud de la asistencia a espacios terapéuticos; (2) expectativas respecto del tiempo libre y actividades de esparcimiento; (3) cambios que, de haber podido, hubieran hecho en el tiempo que los NNDxTEA llevan realizando las terapias; y (4) creencias y pensamientos acerca del futuro del NNDxTEA de parte de sus familiares.

\section{La transformación de las actividades cotidianas, en virtud de la asistencia a espacios terapéuticos}

En consonancia con literatura sobre el tema (Green, 2007; Najar Smeha; Kurtz Cezar, 2011; Schmidt; Dell'Aglio; Bosa, 2007; Smith et al., 2010; Valentine, 2010), una situación frecuentemente mencionada por las madres es que dedican gran parte de su cotidianidad al cuidado y atención de sus NNDxTEA, dentro y fuera del hogar. En las historias de vida, estos cuidados y atenciones implican, entre otras actividades, el acompañamiento y asistencia a múltiples sesiones terapéuticas y controles clínicos que forman parte del diseño terapéutico. Además de la asistencia, las madres se encargan mayoritariamente de la gestión administrativa de los tratamientos y evaluaciones periódicas. Esta abarca, entre otras, la solicitud de turnos en las diferentes especialidades o terapéuticas, renovaciones de autorizaciones para prácticas, y contratación de vehículos privados para los traslados a las sesiones.

Estas actividades además deben diagramarse de manera de no solaparse entre sí y de permitir a la vez tanto la realización de las actividades de los hermanos y hermanas del NNDxTEA (sea acompañándolos a la escuela, dejándolos a cargo del cuidado de adultos, o llevándolos consigo al tratamiento) como de los otros adultos de la familia (padres varones, abuelos y abuelas). De las entrevistadas, solo una manifestó que su esposo también se encargaba de la gestión administrativa de los tratamientos ante las entidades prestadoras de salud.

Las entrevistadas refieren la transformación en la vida cotidiana con posterioridad a la confirmación del diagnóstico, en función de los tratamientos indicados al NNDxTEA.

L: Antes yo tenía la tranquilidad de decir "tengo todo el día libre", digamos, como que era solamente, por ahíde vez en cuando, las reuniones del colegio, llevar al jardín a [otra hija] y lavar la ropa. [...] Y ahora es como que estoy todo el tiempo expectante del horario [...] Tengo que llevar esto, tengo que hacer [...] Ahora como que ¿viste? es una obligación, ¿no? Es como interponer cosas para cumplir con los tres a la vez. Los tres me demandan. Ese es el cambio que yo veo con respecto a mí.

Las actividades narradas por $\mathrm{L}$ y recabadas en todas las entrevistas ilustran de modo emblemático lo que Zerubavel (1981, p. 3) denomina "estructuras secuenciales rígidas” como la forma más obvia de regularidad temporal. Diferentes eventos, actividades y situaciones no pueden por su propia naturaleza llevarse a cabo de modo simultáneo, por lo cual deben ser segregados temporalmente en términos de "antes" y "después". La rigidez y la oscilación entre lo azaroso y lo irreversible son elementos que considerar, y que podemos pensar en estos casos como parte de lo que las madres priorizan o relegan en sus actividades diarias.

Las madres concentran sus actividades habituales en torno a terapéuticas y su gestión administrativa, las tareas de cuidado y las labores domésticas; sin embargo, las primeras funcionan como el ordenador primario de la cotidianidad. Lo expresado en las entrevistas concuerda con la literatura sobre el tema, en lo atinente al lugar prioritario que tiene el cuidado del NNDxTEA en las actividades cotidianas (Najar Smeha; Kurtz Cezar, 2011). 
Abarcando más ampliamente a todos los familiares, se ha marcado que el hecho de que la existencia de un integrante con diagnóstico de autismo en la familia supone una sobrecarga de cuidados para todos los integrantes, en virtud del tiempo y la energía que se vuelcan en relación a la situación (Schmidt; Dell'Aglio; Bosa, 2007) y que se añaden a las otras actividades. En el trabajo de campo observamos, como matiz relevante, que ninguna de las madres entrevistadas manifestó alternancia alguna con actividades sistemáticas, remuneradas o no, por fuera del hogar que no se vinculen al cuidado y acompañamiento del NNDxTEA, algo que también está en consonancia con la literatura sobre el tema (Najar Smeha; Kurtz Cezar, 2011).

Durante la investigación, ninguna dijo tener un empleo formal o informal; hecho que explicaron por la dedicación de tiempo completo a las labores mencionadas. Esta circunstancia coincide con lo que proponen Zanatta et al. (2014) quienes señalan que estos cuidados pueden tornarse arduos y agotadores puesto que implican una dedicación casi exclusiva, a nivel físico y mental (Pozo Cabanillas; Sarriá Sánchez; Méndez Zaballos, 20o6). Según otras investigaciones, esta ausencia de diversificación de las actividades en las que ocupan el tiempo va en detrimento del bienestar psicofísico de las madres (Najar Smeha; Kurtz Cezar, 2011).

Volviendo a las fuentes, L refiere que durante la rutina de cuidados y terapéuticas también se ha sentido agotada, y esto es expresado en varias ocasiones por las otras madres. No obstante, las entrevistadas indican que ellas siempre priorizan el tratamiento. Por ejemplo, M narra con abatimiento y en medio de silencios que, a pesar del cansancio, ella continúa llevando adelante las actividades necesarias para que se realicen los procesos terapéuticos.

Entrevistadora: En todo este proceso ¿Te has sentido alguna vez cansada?

M: Sí, muchas veces. Los primeros días me iba llorando, me iba llorando no sé, de la bronca, no sé, algo... [silencio]... A veces yo llegaba y no quería venir más, pero después me levantaba al otro día y salía de nuevo. Pero es el mismo cansancio... [silencio]... no sé qué debe ser.
Por su parte, V relata entre momentos de risa, aunque con un tono melancólico, que durante el proceso de diagnóstico, y especialmente al inicio de los tratamientos, también se sintió cansada y triste, pero que siempre contó con el acompañamiento de su familia.

Entrevistadora: ¿Cómo te sentías durante ese tiempo? ¿Qué decía tu familia?

V: Cansada, [risas]. Cansada, si... y mi familia, nada. Mi suegro siempre me ayudaba cuando me tenía que traer [al Centro] y, sí, siempre me apoyaron. $Y$ de parte de mi familia también, aunque no los veo muy seguido, pero bueno. Sí, estaba cansada, triste, bueno, pero... luchando.

Esta dedicación intensiva repercute en la crianza de los demás hijos e hijas que no están diagnosticados con TEA. M, con otras dos hijas adolescentes, marcó una mayor independencia en la crianza de esas hijas, y menor tiempo dedicado al cuidado de ellas.

Otro aspecto es la inequidad en la distribución de las labores de cuidado y crianza en el hogar. Mientras las madres entrevistadas se encargan de las tareas descriptas, los padres se dedican a un empleo formal que provea un ingreso económico al hogar. Hernández Bello (2009) indica que la inequidad en la repartición de tiempo, tareas e incluso recompensas, se soporta además en un rol que desde el sector salud se otorga directa o indirectamente a la mujer, lo que facilita la cobertura y eficiencia, disminuye los gastos y transfiere los costos al hogar.

Además los resultados ponen de manifiesto la manera como la vida se transforma y queda subsumida en tratamientos médicos y terapéuticos, lo que permea la subjetividad no sólo de los NNDxTEA, sino también de sus madres. Esta transformación visibiliza una de las modalidades a través de las cuales los procesos de biomedicalización saturan la cotidianidad y la subjetividad, en lo que Clarke y Shim (2011) describen como la realización de la vida y la construcción del sí mismo (self) en función del paradigma biomédico.

En este sentido, un emergente de la investigación es el lugar de hermanos y hermanas de NNDxTEA, 
quienes asisten muchas horas a la semana a espacios terapéuticos en carácter de acompañantes, independientemente de que posean o no, ellos mismos, un diagnóstico psiquiátrico. Estos niños y niñas comparten un espacio y una vida cotidiana en familia impregnados de las recomendaciones, pautas y terapias que los progenitores despliegan con el NNDxTEA. Hemos relevado trabajos enfocados en la importancia de otros familiares, como abuelos (Castro; Piccinini, 2009), y otros que recomiendan realizar investigaciones sobre padres varones (Najar Smeha; Kurtz Cezar, 2011). Consideramos que el emergente antedicho amerita un abordaje más pormenorizado, que tome en cuenta las voces de estos hermanos y hermanas, cuyos tiempos cotidianos y vitales están profundamente incididos por lógicas, parámetros y valores biomedicalizados.

\section{Las expectativas respecto del tiempo libre, las actividades de esparcimiento y la responsabilización por la salud}

Frente a la pregunta acerca de la dedicación a actividades diferentes a las terapéuticas, algunas madres se mostraron sorprendidas, como si se les recordara la posibilidad. En ningún caso refirieron asistencia sistemática o regular a actividades artísticoculturales, deportivas, recreativas, de esparcimiento individual u otras acciones ligadas a las relaciones de socialización o afectivas no asociadas al cuidado del NNDxTEA. Sólo en un caso hubo referencia a la práctica de un deporte, y de manera esporádica.

Este hecho se liga con los hallazgos del apartado anterior, ya que el predominio de actividades vinculadas a las terapias y el cansancio manifestado conllevan una sobrecarga respecto a sus rutinas cotidianas. La implicación de las madres en las actividades, además, se evidencia que ellas asumen la responsabilidad por la salud de los NNDxTEA a tiempo completo.

Y: Sí, [desde el diagnóstico] cargué con mucha responsabilidad yo sola. Vamos a decir que, como que se te posterga la vida, porque hoy tenés que meterle a las terapias del nene. Bueno es una mamá que piensa así, como que el nene me necesita, el nene va a tener terapia, y en mí no pienso.
L: Digamos antes del diagnóstico era levantarme a cualquier hora, sabía que tenía todo el día libre [...] $Y$ que [era] no tener horario porque digamos era [la otra hija] que iba al colegio, la llevaba y volvía, seguía rutina normal. Iba a la casa de mi hermana [a] tomar unos mates... ahora mi hermana me dice "Tomá unos mates". Le digo "Mirá, puedo ir media hora, más no; tengo que hacer esto, tengo que hacer aquello, tengo que ir con T para allá, tengo que llevarlo...".

Estos sentimientos de responsabilidad hacia la salud del NNDxTEA se enmarcan como otra característica de los procesos de biomedicalización. La salud se erige como una obligación moral, predominantemente individual, y entronca con la rutinización de la intervención biomédica (Clarke et al., 2010). Estar y permanecer saludable se aplica también a los padecimientos crónicos, mediante el principio de optimización, que exhorta no sólo a procurar sanar lo más rápido posible de una enfermedad, sino también a "administrar apropiadamente la enfermedad crónica” (Clarke et al., 2010, p. 63). Esta exhortación tiene un correlato cultural más amplio, que excede a quienes tienen un diagnóstico medicalizado, y que podemos identificar en las madres que están a cargo de sus NNDxTEA. Este correlato consiste en estar en permanente actividad, mantenerse saludable, y cumplir con los mandatos de autorregulación, una empresa en la que precisamente "no hay descanso para los cansados” (Clarke et al., 2010, p. 63).

Otro aspecto relacionado con el tiempo libre y las actividades de esparcimiento atañe a los vínculos sociales y de afecto. Respecto de las actividades ligadas a las relaciones de socialización o afectivas que no estén asociadas al cuidado del NNDxTEA, las entrevistadas han referido principalmente el encuentro con familiares. Se han mencionado padres, abuelos y abuelas, tías, hermanos y hermanas del NNDxTEA cuando aluden a las actividades cotidianas vinculadas a la continuidad terapéutica. No en todos los casos esa referencia se dio en el marco de considerarlos apoyos o auxilios explícitos en las rutinas. Es significativo que las entrevistadas no mencionaron a amigas mujeres en ningún caso, y que hubo alusiones marginales a otros vínculos de tipo técnico, como los profesionales de la salud o la educación. Únicamente en un caso se aludió a la 
figura de la maestra integradora con rol de ayuda y acompañamiento, a fin de preparar al niño para el ingreso al jardín de infantes. En trabajos sobre el tema, se ha señalado que las madres que poseen más apoyos y los perciben como más útiles presentan menores niveles de estrés (Pozo Cabanillas; Sarriá Sánchez; Méndez Zaballos, 20o6).

Por último, tampoco documentamos una vinculación de tipo religiosa que oficie de amparo, sostén o acompañamiento, ni de adaptación a la situación, algo que en investigaciones es señalado como un modo que las madres encuentran para aliviar la angustia y la incerteza por la situación (Najar Smeha; Kurtz Cezar, 2011; Núñes, 2007; Welter et al., 2008).

En línea con los aportes de Rabinow, diversos autores sostienen que los procesos de biomedicalización promueven la formación de biosocialidades (Bell; Figert, 2015; Clarke et al., 2010; Gibbon; Novas, 2008). Sin embargo, en ningún caso, las entrevistadas manifestaron dedicar tiempo a participar de espacios individuales o colectivos en los que intercambien experiencias, compartan dudas, expectativas o angustias, o adquieran conocimientos acerca de acciones colectivas en pos de mejorar, dar continuidad o garantizar los tratamientos para NNDxTEA. Esta inexistencia de una red de contención y apoyo sugiere una fragilidad mayor de las madres, según Najar Smeha y Kurtz Cezar (2011).

En Argentina las dos principales agrupaciones de familiares de NNDxTEA son Padres Autoconvocados con Hijos con Autismo (TGD-Padres TEA, http://tgdpadres.com.ar/) y Asociación Argentina de Padres de Autistas (APADeA, http://apadea.org.ar/), con presencia en los medios y en las redes sociales. Ambas llevan adelante una nutrida agenda de actividades de concientización, talleres y cursos de formación y asesoramiento, y eventos en la vía pública, con el Día Nacional de Concientización del Autismo como punto destacado. Además, en 2014 se sancionó en Argentina la Ley 27043, Ley Integral de Trastornos del Espectro Autista (TEA), con apoyo del Ministerio de Salud, la Sociedad Argentina de Pediatría, la Sociedad de Neurología Infantil y la Asociación Argentina de Psiquiatría Infanto-Juvenil. La ley (que aún no fue reglamentada) sostiene la necesidad de un abordaje integral e interdisciplinario de los
TEA, y obliga a los agentes de salud -obras sociales, empresas de medicina prepaga y organizaciones de la seguridad social- a brindar prestaciones para el estudio, la detección temprana, el diagnóstico y el tratamiento de los TEA. En ninguna instancia de las entrevistas, las madres refirieron estar al tanto de las asociaciones de padres ni de la entonces recientemente sancionada ley nacional y los apoyos institucionales recibidos.

Estas consideraciones van en consonancia con Kuhaneck et al. (2010), quienes reportaron que las madres de niños con diagnóstico de TEA refieren que el tiempo con los NNDxTEA es intenso y extenuante emocional y físicamente. De allí la necesidad de las madres de contar con lo que llaman "tiempo para mí" (Kuhaneck et al., 2010, p. 344), y describen diversas actividades que realizan para obtenerlo: ejercicios físicos, reunirse con amigas, hacer compras, ir a almorzar con amigas y pasar tiempo con los otros hijos e hijas que no tienen diagnóstico. Para las madres cuyas historias de vida presentamos aquí, muchas de estas opciones no están formuladas ni siquiera como una fantasía.

Como corolario de la dedicación integral al cuidado del NNDxTEA, entonces, encontramos una restricción respecto de otras actividades ligadas tanto al ocio creativo como al activismo colectivo. Del análisis podemos inferir que existe un modelamiento de la subjetividad de las madres, de las relaciones familiares y de los tiempos cotidianos, en clave de biomedicalización de la vida. Sin embargo, este modelamiento no deriva de acciones conjuntas o encuentros con otras madres que atraviesan situaciones análogas, ni de una participación más amplia en agrupaciones que aglutinan demandas en relación a la cobertura de tratamientos de los NNDxTEA, que no son referidas aunque ya existían en el momento de las entrevistas.

\section{Los cambios que, de haber podido, hubieran hecho en el tiempo que los NNDXTEA Ilevan realizando las terapias}

Este eje se vincula a un ejercicio en el que se planteó, como interrogante, qué cambios realizarían las madres en su vida cotidiana o en todo el proceso que se relaciona con los NNDxTEA. Todas las madres 
coincidieron en indicar que sí, realizarían algún tipo de cambio. Algunas indicaron que cambiarían la distribución de tareas en el hogar.

Entrevistadora: Sí, vos lo traes a la casa, [el padre de I] trabaja, las nenas hacen una cosa ¿Cambiarías algo?

L: Sí [risas]. Poder tener un poco de tiempo para mí. Sí, porque ahora yo estoy prácticamente para ellos, nada más...

Entrevistadora: Si te dieran una varita mágica y te dijeran "podés cambiar". ¿Qué cambiarías?

L: De novenir más sesiones a la terapia. A veces uno dice ¿por qué no nos podemos quedar en casa? De estar iviste? Hacer, dentro de todo, hacer como se dice, una "vida normal". Ir a la plaza... que por ahí no podemos hacer eso. Ehhh, ya no hacemos tantas salidas en familia [...]. Yo digo, porque por ahí el poco tiempo que estoy en casa, quiero descansarnada más. No me da para sacar a la plaza a los chicos, y por ahí cambiaría eso. Tener más tiempo como familia y no tantas cosas para hacer. Tanta obsesión por el horario, tal horario esto, tal horario aquello. ¿Viste? Ya llega un horario, ya tengo que tener la comida lista porque ya después me tengo que ir. Eso cambiaría.

Lo expresado por L pone en escena otra faceta de la regularidad temporal trabajada por Zerubavel (1981, p. 5-6), la "duración rígida”, que alude al hecho de que numerosos eventos están asociados a duraciones relativamente rigurosas. La mayoría de los cronogramas y programaciones de horarios son posibles porque se produce una rigidificación de la duración de los eventos que la componen. También marca que las duraciones rígidas están condicionadas por cuestiones tanto biológicas como tecnológicas, que muchas veces son vivenciadas como intrínsecas a la actividad.

Por otra parte, algunas entrevistadas indicaron que el cambio deseado en su cotidianidad estaría orientado a cambiar el diagnóstico, como resultado de la cura.

Y: No. No sé.... porque realmente te diría que mi hijo se cure, pero [aunque] a mí me venga una pastilla de Estados Unidos -porque la pastilla que cura el autismo va a venir acá, con mucha fe va a venir acá- mi nene no se va a curar. Porque por el tema que le faltan cositas en la cabeza. Pero bueno, pero ya está todo organizado, así que como para que... tiene todo como para él salir adelante. Para que él salga adelante, hable... Todo.

Entrevistadora: ¿Cambiarías algo de todo este proceso? ¿Cambiarías algo en este momento?

V: ¿Del proceso, o del diagnóstico? [risas]. Que no existiera ese diagnóstico.

\section{Entrevistadora: ¿Y del proceso?}

V: No, no porque ellos evolucionaron y bueno, es lo... lo que mejor se puede.

Entrevistadora: Idealmente, si te dieran una varita mágica, $M$ ¿qué cambiarías por ejemplo?

M: [Lo cambiaría] a $T$ [el hijo con diagnóstico de TEA], lo único. Lo curaría y ya está. Después...

Como mencionamos, las madres denotan cansancio y sobrecarga, pero además existe una resignación que gira en torno a una cuasi predestinación mediada por el diagnóstico.

Y: Me costó todo, mucho, pero ya todo está cavilado. Pero si, yo lo primero [es que] me sentía muy mal porque te decís por qué, por qué, por qué, pero no le encontrás el porqué de nada. Tenés que darle para adelante, pero bueno. Ahora de todo hay que aprender.

Distintas investigaciones documentaron que las madres de NNDxTEA experimentan emociones tales como tristeza, incertidumbre, inconformismo, culpa (Welter et al., 2008) y también shock, desilusión, ansiedad, preocupación, especialmente al recibir la confirmación diagnóstica (Schmidt; Bosa, 2007; Schmidt; Dell'Aglio; Bosa, 2007). Sin embargo, Green (2007, p. 155) documentó en su estudio que las entrevistadas describen un momento decisivo y consciente, que se ubica relativamente cerca del momento del diagnóstico, en el que se sienten 
habilitadas para ir más allá de la pena y dar continuidad al cuidado del hijo o hija.

De todos modos, un emergente de este eje es que, aunque dan cuenta de una cierta adaptación a la lógica biomedicalizada, persiste una tensión consistente en que, si pudieran, se retirarían de esa dinámica. En términos de identidad, esta constatación va en línea con lo expresado por Hall, quien plantea que "las identidades nunca se unifican y [...] están cada vez más fragmentadas y fracturadas; nunca son singulares, sino construidas de múltiples maneras a través de discursos, prácticas y posiciones diferentes, a menudo cruzados y antagónicos" (Hall, 2003, p. 17).

Si consideráramos a la biomedicalización como un espacio, diríamos que en las historias de vida de las madres entrevistadas se puede identificar una aparente contradicción, de saturación y vacío a la vez. Por un lado, la biomedicalización está implícita, es una lógica que permea casi todos los aspectos de la vida cotidiana de estas madres, colmando sus actividades y organizando su tiempo, y en gran medida el de las familias. Por el otro, las entrevistadas no refirieron vinculación con otras madres o familias de NNDxTEA. Este constituye el vacío de vínculos que coexiste con la saturación del tiempo biomedicalizado. Este emergente marca una línea diferente para considerar respecto de otras investigaciones que analizan a progenitores de NNDxTEA en particular y, más ampliamente, a grupos de apoyo en relación a diagnósticos medicalizados (Nunes; Ortega, 2016).

\section{La biomedicalización y el futuro}

El interrogante por el futuro fue el más difícil de responder para las entrevistadas; en general requirió más tiempo para obtener la respuesta y puso en evidencia sentimientos de incertidumbre. Esto en algún caso derivó en expresiones de tristeza, provocando llanto y mucha congoja, y en otros tuvo menor carga emotiva, e incluso ameritó algunas risas, no exentas de tensión o nerviosismo.

Entrevistadora: ¿Qué pensás, $Y$, del futuro de A? ¿Alguna vez te lo has planteado?

Y: No. Quiero, que ahora va al colegio especial, llegar a que se sepa valer por sí solo. Después que él sea... capaz que en el colegio le puedan ofrecer algo, pero no sé.

Entrevistadora: ¿Cómo te pensás que pueda ser el futuro con B? ¿Alguna vez te lo has planteado?

V: Y, vamos a ver. Ehh según cómo evolucione, quizá puede ser que sea muy duro, quizá puede ser más llevadero, no sé todavía [risas].

Entrevistadora: Ahora te pregunto. ¿Han pensado en algún momento el futuro de I y la familia?

L: No. Digamos, yo la vista al futuro es "el año que viene". [Tengo que] buscar un colegio para él. Más de eso no, porque yo sé que él es muy cambiante. Digamos, yo puedo proponer todo, pero el que dispone es él, y está todo a medida del tiempo de él. Yo séque él... yo no quiero tener más perspectivas de lo que él pueda llegar a dar, entonces voy paso a paso. Por ahí, no te niego, me agarra la ansiedad digamos de "¿Por qué no hablás I? Ya tenés que hablar". "Dale, vos podés hablar". Pero yo sé que él tiene su tiempo. Cuando él pueda y quiera hablar, lo va a hacer. Me agarra como la ansiedad de decir: “ ¿Si hace ya cuánto que estamos con el tratamiento? Ya tenés que hablar".

Entrevistadora: ¿Cómo se ven el futuro con T? ¿Alguna vez lo han pensado, se lo han planteado?

M: No sé, la verdad [es] que no quiero ni pensarlo.

Entrevistadora: ¿El papá te lo ha planteado en algún momento?

M: Él siempre dice que... [Negación gestual, llanto]

Entrevistadora: Tranquila ¿Pasamos a la siguiente pregunta?

\section{M: [Afirmación gestual]}

Esta dimensión de análisis puede ser abordada desde múltiples direcciones, sin embargo, a los efectos de este artículo nos interesa marcar los emergentes que se vinculan con los demás ejes trabajados. Así, una primera constatación surgida 
desde las historias de vida consiste en la dificultad para imaginar un futuro, la congoja que puede provocar, y cómo el tiempo futuro tanto de ellas como de los NNDxTEA está marcadamente incidido por el diagnóstico.

Otro emergente, congruente con lo expresado por Green (2007), consiste en que, aunque los tratamientos prescriptos para niños con alguna discapacidad insumen gran cantidad de tiempo y trabajo para sus madres, la mayoría de las participantes de su estudio refirieron su certeza en la importancia de esos tratamientos y en la necesidad de luchar por mantenerlos.

Más ampliamente, es significativo que, frente a la pregunta por el futuro, ninguna de las participantes lo imagina junto con otras personas que atraviesen situaciones similares. En este punto, es importante no asumir que el agrupamiento con otros progenitores sería la trayectoria más deseable o la mejor posible, ni que funcionaría invariablemente como aliviadora de las situaciones antes referidas. Al respecto, vale destacar el trabajo de Kuhaneck et al. (2010) en el que las madres entrevistadas, que asistían a grupos de apoyo, refirieron que su inclusión en los mismos no resultó efectiva como estrategia para afrontar la situación, ni para mejorar su salud mental. De modo que las biosocialidades no sólo se ponen en juego en los grupos de pares, y las identidades biomedicalizadas pueden modelarse con prescindencia de estos agrupamientos.

\section{Conclusiones}

Dado el carácter cualitativo de la metodología en la que se sustenta este artículo, y el hecho de estar basado en cuatro historias de vida, las conclusiones no apuntan a expresar tendencias mayoritarias, ni ser representativas de las madres y familiares de NNDxTEA. Antes bien, nos interesa marcar líneas en curso y en tensión, y la importancia de avanzar en otras, apenas esbozadas en las entrevistas.

Al nivel micro de la biomedicalización, analizamos las relaciones y situaciones cotidianas que contribuyen a la medicalización de los NNDxTEA y los tiempos y dinámicas familiares de las entrevistadas, en función de la participación directa en los contextos clínicos y terapéuticos tanto del NNDxTEA como del resto de la familia. En este punto los resultados incluyen: (1) La organización de los tiempos y dinámicas familiares en función de los tratamientos; (2) La transformación de pautas respecto a la crianza y el cuidado del NNDxTEA, que también permea a sus hermanos y hermanas; (3) El sentimiento de responsabilidad y continua vigilancia de la salud, de parte de las madres hacia el NNDxTEAy, en menor medida e instrumentalmente, de ellas mismas; (4) La transformación subjetiva e identitaria en función del diagnóstico; y (5) La fortísima dependencia de los servicios terapéuticos.

Derivado de esto, documentamos además una doble vinculación con el tiempo de parte de las madres entrevistadas. Por un lado, no se dan "tiempo para sí”, abocadas como están a la permanente e incesante tarea de brindar cuidados a sus NNDxTEA. Por el otro, y como corolario de lo primero, tampoco emplean tiempo en inscribirse, siquiera a modo de fantasía, en un colectivo con otras personas que atraviesan su misma situación.

Sobre lo primero, algunos autores lo consideran como un problema grave para la salud mental de las madres, y sostienen que la psicología puede funcionar como red de apoyo a estas mujeres y contribuir para la prevención en salud mental en las familias que poseen un integrante con diagnóstico de autismo, y que es necesario posibilitar a estas madres un espacio donde ellas puedan ser escuchadas, intercambiar experiencias y amenizar sus incertidumbres (Najar Smeha; Kurtz Cezar, 2011). Sobre lo segundo, esta circunstancia podría estar dando la pauta de lo incipiente que es en Argentina la incidencia de los grupos de padres de niños con TEA, a diferencia de lo relevado en países como Brasil (Nunes; Ortega, 2016). Finalmente, lo documentado da una pauta de identidades biomedicalizadas y biosocialidades, que, lejos de estructurarse como destino inexorable, se configuran en permanente tensión.

\section{Referencias}

APA - AMERICAN PSYCHIATRIC ASSOCIATION. Diagnostic and statistical manual of mental disorders. 5. ed. Washington, DC: APA, 2013.

ARGENTINA. Ministerio de Salud. Resolución $n^{0}$ 1.480/2011. Guía para investigaciones en salud 
humana. Buenos Aires: Ministerio de Salud, 2011. Disponível em: <https://bit.ly/2R23gJJ>. Acesso em: 23 dez. 2017.

BELL, S. E.; FIGERT, A. E. Reimagining (bio) medicalization, pharmaceuticals and genetics: old critiques and new engagements. New York: Routledge, 2015.

CASTRO, E.; PICCININI, C. Implicações da doença orgânica crônica na infância para as relações familiares: algumas questões teóricas. Psicologia: Reflexão e Crítica, Porto Alegre, v. 15, n. 3, p. 625635, 2009.

CLARKE, A. E.; SHIM, J. Medicalization and biomedicalization revisited: technoscience and transformations of health, illness and American medicine. In: PESCOSOLIDO, B. A. et al. (Ed.). Handbook of the sociology of health, illness, and healing: a blueprint for the 21st Century. New York: Springer. 2011. p. 173-195.

CLARKE, A. et al. Biomedicalization: technoscience, health and illness. Durham: Duke University Press, 2010.

CONRAD, P. The medicalization of society: on the transformation of human conditions into treatable disorders. Baltimore: The John Hopkins University Press, 2007.

CONRAD, P. Medicalization: changing contours, characteristics, and contexts. In: COCKERHAM, W. Medical sociology on the move: new directions in theory. New York: Springer, 2013. p. 195-214.

CONRAD, P.; POTTER, D. From hyperactive children to ADHD adults: observations on the expansion of medical categories. In: CONRAD, P.; LEITER, V. (Ed.). Health and health care as social problems. New York: Rowman \& Littlefield, 2003. p. 39-65.

CONRAD, P.; SCHNEIDER, J. W. Looking at levels of medicalization: a comment on Strong's critique of the thesis of medical imperialism. Social Science \& Medicine, Amsterdam, v. 14, n. 1, p. 75-79, 1980.

FLAHERTY, M. A watched pot: how we experience time. New York: New York University Press, 1999.
FLICK, U. Introducción a la investigación cualitativa. Madrid: Morata, 2004.

FRITH, U. Autismo: hacia una explicación del enigma. Madrid: Alianza, 1992.

GIBBON, S.; NOVAS, C. Biosocialities, genetics and the social sciences: making biologies and identities. London: Routledge, 2008.

GREEN, S. E. "We're tired, not sad": benefits and burdens of mothering a child with a disability. Social Science \& Medicine, Amsterdam, v. 64, n. 1, p. 150-163, 2007.

HALFMANN, D. Recognizing medicalization and demedicalization: discourses, practices, and identities. Health, London, v. 16, n. 2, p. 186-207, 2012.

HALL, S. Introducción ¿Quién necesita identidad? In: HALL, S.; DU GAY, P. (Org.). Cuestiones de identidad cultural. Buenos Aires: Amorrortu, 2003. p. 13-39.

HERNÁNDEZ BELLO, A. El trabajo no remunerado de cuidado de la salud: naturalización e inequidad. Gerencia y Políticas de Salud, Bogotá, v. 17, n. 8, p. 173-185, 2009.

HERNÁNDEZ SAMPIERI, R.; FERNÁNDEZCOLLADO, C.; BAPTISTA LUCIO, P. Metodología de la investigación. Ciudad de México: McGraw Hill Interamericana, 2006.

KANNER, L. Trastornos autistas del contacto afectivo. Revista Española de Discapacidad Intelectual Siglo Cero, Salamanca, n. 149, p. 1-36, 1993.

KUHANECK, H. M. et al. A qualitative study of coping in mothers of children with an autism spectrum disorder. Physical \& Occupational Therapy in Pediatrics, London, v. 30, n. 4, p. 340-350, 2010.

MALLIMACHI, F.; GIMÉNEZ VÉLIVEAU, V. Historia de vida y métodos biográficos. In: VASILACHIS DE GIALDINO, I. (Coord.) Estrategias de investigación cualitativa. Barcelona: Gedisa, 2006. p. 171-212.

MARRADI, A.; ARCHENTI, N.; PIOVANI, J. I. Metodología de las ciencias sociales. Buenos Aires: Emecé, 2007. 
NAJAR SMEHA, L.; KURTZ CEZAR, P. A vivência da maternidade de mães de crianças com autismo. Psicologia em Estudo, Maringá, v. 16, n. 1, p. 43-5o, 2011.

NÚÑES, B. A. Familia y discapacidad: de la vida cotidiana a la teoría. Buenos Aires: Lugar, 2007.

NUNES, F.; ORTEGA, F. Ativismo político de pais de autistas no Rio de Janeiro: reflexões sobre o "direito ao tratamento". Saúde e Sociedade, São Paulo, v. 25, n. 4, p. 964-975, 2016.

PARIS, J. The ideology behind DSM-5. In: PARIS, J.; PHILLIPS, J. (Ed.). Making the DSM-5: concepts and controversies. New York: Springer, 2013. p. 39-46.

POZO CABANILLAS, P.; SARRIÁ SÁNCHEZ, E.; MÉNDEZ ZABALLOS, L. Estrés en madres de personas con trastornos del espectro autista. Psicothema, Oviedo, v. 18, n. 3, p. 342-347, 2006.

RABINOW, P. Essays on the anthropology of reason. New Jersey: Princeton University Press, 1996.

SANDOVAL, C. Investigación cualitativa: Instituto Colombiano para el Fomento de la Educación Superior. Bogotá: Arfo, 2002.

SCHMIDT, C.; BOSA, C. A. Estresse e auto-eficácia em mães de pessoas com autismo. Arquivos Brasileiros de Psicologia, Rio de Janeiro, v. 59, n. 2, p.179-191, 2007.

SCHMIDT, C.; DELL'AGLIO, D.; BOSA, C. A. Estratégias de coping de mães de portadores de autismo: lidando com as dificuldades e com a emoção. Psicologia: Reflexão e Crítica, Porto Alegre, v. 20, n. 1, p. 124-131, 2007.

SMITH, L. E. et al. Daily experiences among mothers of adolescents and adults with autism spectrum disorder. Journal of Autism and Developmental Disorders, New York, v. 40, n. 2, p. 167-78, 2010.

VALENTINE, K. A consideration of medicalisation: choice, engagement and other responsibilities of parents of children with autism spectrum disorder. Social Science \& Medicine, Amsterdam, v. 71, n. 5, p. 950-957, 2010.
VALLES, M. Técnicas cualitativas de investigación social: reflexión metodológica y práctica profesional. Madrid: Síntesis, 2000.

WELTER, I. et al. Gênero, maternidade e deficiência: representação da diversidade. Revista Textos e Contextos, Porto Alegre, v. 7, n. 1, p. 98$119,2008$.

ZANATTA, E. et al. Cotidiano de famílias que convivem com o autismo infantil. Revista Baiana de Enfermagem, Salvador, v. 28, n. 3, p. 271-282, 2014 .

ZERUBAVEL, E. Hidden rythms: schedules and calendars in social life. Berkeley: University of California Press, 1981.

ZERUBAVEL, E. Time maps: collective memory and the social shape of the past. Chicago: The University of Chicago Press, 2004.

\section{Contribución de los autores}

Las autoras participaron juntas en todas las instancias de elaboración del artículo, desde la concepción, el análisis e interpretación de los datos, hasta la redacción y revisión crítica.

Recibido: $29 / 12 / 2017$

Aprobado: $11 / 12 / 2018$ 